\title{
Volunteer First Responders for Optimizing Management of Mass Casualty Incidents
}

Eli Yafe, PhD; Blake Byron Walker, PhD; Ofer Amram, PhD; Nadine Schuurman, PhD; Ellen Randall, MSc; Michael Friger, PhD; Bruria Adini, PhD

\section{ABSTRACT}

Objective: Rapid response to a trauma incident is vital for saving lives. However, in a mass casualty incident $(\mathrm{MCl})$, there may not be enough resources (first responders and equipment) to adequately triage, prepare, and evacuate every injured person. To address this deficit, a Volunteer First Responder (VFR) program was established.

Methods: This paper describes the organizational structure and roles of the VFR program, outlines the geographical distribution of volunteers, and evaluates response times to $3 \mathrm{MCls}$ for both ambulance services and VFRs in 2000 and 2016.

Results: When mapped, the spatial distribution of VFRs and ambulance stations closely and deliberately reflects the population distribution of Israel. We found that VFRs were consistently first to arrive at the scene of an $\mathrm{MCl}$ and greatly increased the number of personnel available to assist with $\mathrm{MCl}$ management in urban, suburban, and rural settings.

Conclusions: The VFR program provides an important and effective life-saving resource to supplement emergency first response. Given the known importance of rapid response to trauma, VFRs likely contribute to reduced trauma mortality, although further research is needed in order to examine this question specifically. (Disaster Med Public Health Preparedness. 2019;13:287-294)

Key Words: mass casualty incidents, volunteers, response times, triage, emergency medical services

\section{INTRODUCTION}

Mass casualty incidents (MCIs) are events in which a large volume, variety, and/or severity of trauma casualties occur, potentially overwhelming the health care capacity within a given community. ${ }^{1-4}$ Due to the sudden demand for emergency response, triage, evacuation, and trauma care, an MCI strains the balance between existing resources and those required to address the incident. ${ }^{2-5}$ A trauma system's ability to successfully manage an MCI therefore rests on its ability to rapidly adjust resources to meet the specific and unique demands of a given $\mathrm{MCI}$ event. ${ }^{6}$ Maintaining this adaptability poses a significant challenge meriting close examination to maximize the life-saving potential of a given response system.

Managing an $\mathrm{MCI}$ at the prehospital stage is one of the most challenging and complex issues faced by emergency medical systems (EMS). ${ }^{7,8}$ The deployment and delivery of prehospital care at the scene of the incident relies on triaging-a process of prioritizing, organizing, and delivering care in a manner that makes the most efficient and effective use of available resources. ${ }^{9-11}$ Depending on how MCIs are handled, they can demonstrate the effectiveness of a well-organized system of delivery of care or, conversely, lapse into a chaotic situation that overwhelms emergency services both at the scene and at the receiving hospitals. A critical component of successful $\mathrm{MCI}$ management is ensuring that personnel and resources arrive on the scene in a timely manner to administer care as necessary and effect a rapid evacuation of the wounded. ${ }^{12}$ In a country that contends with warfare- and terror-related MCIs, the Israeli emergency first response services rely on maintaining a trauma system that has the capacity to rapidly deploy resources available for a first response. They have developed a unique first responder system that draws on volunteers who are geographically distributed across the country. This system serves both as an effective MCI response tool and as a potential exemplar or test case for establishing volunteer-based emergency response systems in other countries.

Magen David Adom (MDA) is Israel's primary emergency first response service, ${ }^{13}$ operating across the country and comprising both employees and volunteers. ${ }^{14}$ MDA's service covers approximately 28600 square kilometers and services over 8 million people. In the beginning of the millennium (2000), MDA implemented a special volunteer force of on-call first responders to assist in the delivery of care at the prehospital stage. The primary goal of this Volunteer First Responders (VFR) program is to 
significantly shorten ambulatory response time to both $\mathrm{MCI}$ and routine individual patients (eg, cases of cardiac arrest and falls). The mechanism to realize this goal is the deployment of on-call volunteers equipped with life support equipment, in tandem with the standard MDA ambulance team, ${ }^{15}$ with the intent that VFR members reach victims before the ambulance arrives, begin treatment, inform and guide the EMS teams, and assist in EMS treatment thereafter. The MDA VFR now comprises approximately 3500 adult volunteers with advanced training in emergency medicine across Israel. These volunteers are geographically distributed throughout the country in order to effectively deploy within a few minutes whenever and wherever an incident occurs.

This paper provides an assessment of the potential added value provided by the VFR program, first through an overview of MDA MCI management and the VFR program, followed by a quantitative comparison of call-to-arrival times for VFRs and regular MDA personnel. We analyzed data from $3 \mathrm{MCI}$ in Israel: 2 that occurred towards the end of year 2000, when the VFR program was initiated, and a third that occurred in mid-2016.

\section{Mass Casualty Incidents}

A MCI is an incident in which the number of casualties exceeds the standard capacity of the emergency medical services to provide care. ${ }^{16}$ The spatial and temporal unpredictability of $\mathrm{MCI}$ pose significant challenges in $\mathrm{MCI}$ response planning. ${ }^{17}$ First responders' time from the call to arrival on scene and the time from the scene to patient arrival at a trauma center are critical elements for optimizing patient outcome. In addition to the time and location of the MCI, the number of victims, severity of injuries, characteristics of the geographic location, risk of response, and duration of the event are all known factors in patient outcome. ${ }^{12,18}$

At the patient level, medical differences between routine calls and MCI response can be significant. In a routine or isolated incident, standard EMS resources are typically sufficient to enable EMS teams to successfully stabilize a patient prior to evacuation. In contrast, the relatively limited number of EMS responders and medical equipment restricts the provision of care to multiple patients in an MCI. Therefore, on-scene response to MCIs must focus on vital life-saving operations and triage of casualties according to injury severity.

Key principles of MCI management include response command and information gathering (eg, exact location, individual risk identification, access and evacuation routes, casualty counting, and casualty ranking by urgency of their medical situation). Postresponse tasks include calling for additional personnel, finding access routes, contacting hospitals, and preparing hospitals to receive casualties. ${ }^{19,20}$ The VFR program was initiated with the belief that that many of these tasks could be delegated to trained volunteers to increase professional first responders' time and resources on-scene.

\section{Management}

In Israel, the MDA manages prehospital care both on-scene and during patient transport to a trauma center or hospital. ${ }^{13}$ Due to a relatively high number of MCIs, the MDA has developed an approach to incident response that follows the general principles of triage, with two caveats: MCIs often require modifications to triage protocols, ${ }^{21}$ and treatment and evacuation decisions must often be made on-scene.

The immediate task of MDA first responders is to triage casualties according to injury severity. ${ }^{22}$ Patients with lifethreatening injuries will be prioritized above those with mild injuries may; those with mild injuries may experience delayed care due to personnel and equipment shortages. If the situation permits, severely injured casualties will be ranked by urgency in order to optimize response efficiency. ${ }^{23}$

Patient evacuation is dependent on personnel and ambulances available on scene or nearby, though all available means (eg, volunteer vehicles and private vehicles) should be used where possible. Patients are most often evacuated to a designated trauma center, though the distribution of patients among multiple hospitals is preferable to avoid case overload in any single trauma unit. However, responders must decide for each patient whether to stabilize and treat them on-scene or evacuate them immediately to a trauma center.

\section{The MDA VFR Program}

The management and handling of MCIs is directly affected by the distance from the event to the nearest capable medical center. ${ }^{17}$ The successful management of MCIs depends on a timely first response, which is critically affected by the distance of the first responders to the event scene. This is particularly relevant for MCIs that occur more than 15 kilometers outside of an urban area. These events, classified as rural MCIs, can have incident-to-first response times of more than 10 minutes, a medically significant duration in many cases. The development of the VFR program was based on the premise that mobilizing local volunteers, traveling on foot or in personal vehicles, could reduce the critical interval of time for getting first responders to the scene.

MDA began by recruiting approximately 1000 volunteers who were senior graduates of specialized emergency medicine courses. All volunteers now undergo the Prehospital Trauma Life Support training course to ensure uniform professional standards in ambulatory care delivery throughout the trauma system. ${ }^{22}$ They carry emergency medical equipment and are either continuously listening to MDA's control networks or are called via pager or mobile phone. The MDA uses this system to deploy the VFR to thousands of routine life-saving operations each month.

\section{DATA AND METHODS}

This study was approved by the Research Ethics Committee of the MDA. To assess the utility of the VFR program, we reviewed 3 similar MCIs occurring in 3 different geographical 


\section{Description of the $3 \mathrm{MCls}$}

Location

Seven Stars Interchange

City of Haifa

Mitzpe Jericho
Geography

Suburban

Urban

Rural
Date

09/29/2000

$11 / 22 / 2016$

$12 / 13 / 2000$
Cause

Bus rollover

Bus rollover

Bus rollover
No. Casualties

49

51

22

TABLE 2

Number of Volunteer First Responders (VFRs) and Magen David Adom (MDA) Ambulance Stations Within 1, 5, 10, and 20 $\mathrm{km}$ of Each Incident, and the Distance From Each Incident to the Nearest VFR Residence and MDA Station

\begin{tabular}{|c|c|c|c|c|c|c|}
\hline & & $1 \mathrm{~km}$ & $5 \mathrm{~km}$ & $10 \mathrm{~km}$ & $20 \mathrm{~km}$ & Nearest $(\mathrm{km})$ \\
\hline \multirow[t]{2}{*}{ Seven Stars Interchange (suburban) } & VFR residence & 0 & 11 & 77 & 365 & 3.2 \\
\hline & MDA ambulance station & 0 & 1 & 5 & 11 & 4 \\
\hline \multirow[t]{2}{*}{ Haifa (urban) } & VFR residence & 10 & 86 & 114 & 217 & 0.9 \\
\hline & MDA ambulance station & 0 & 2 & 3 & 8 & 4.5 \\
\hline \multirow[t]{2}{*}{ Mitzpe Jericho (rural) } & VFR residence & 0 & 0 & 0 & 9 & 10.9 \\
\hline & MDA ambulance station & 0 & 0 & 0 & 3 & 10.9 \\
\hline
\end{tabular}

settings (urban, suburban, and rural). Each MCI had at least 20 patients. Two MCIs (the Seven Stars Interchange and Mitzpe Jericho) occurred near the establishment of the VFR program, while the most recent event occurred in 2016, long after the program's inception. We disregard the cause of the MCI in this analysis. The $3 \mathrm{MCI}$ are described in Table 1 .

Detailed administrative data for each incident were acquired from MDA. The time from initial reporting of the incident until each responder's arrival on scene were categorized into 5-minute intervals for both VFR and MDA personnel.

The geographical location of the incident and the location of VFR residences were also collected and mapped using Geographical Information Systems. To provide an estimate of resource access and availability, the number of VFRs within a distance of $2 \mathrm{~km}$, $5 \mathrm{~km}, 10 \mathrm{~km}$, and $20 \mathrm{~km}$ from each incident was calculated, as was the distance from each incident to the nearest VFR.

\section{RESULTS}

The geographical distribution of VFRs and MDA ambulance stations reflects the population distribution of Israel, with the majority of volunteers residing along the Mediterranean coastline in the cities of Tel Aviv and Haifa (Figure 1). In the vicinity of Mitzpe Jericho (east of Jerusalem) and the Negev (the southern section of the study area), relatively few volunteers and MDA stations are situated, as these regions are predominantly desert with a very low population density.

While the urban MCI location had 10 volunteers within a $1-\mathrm{km}$ radius, both the suburban and rural had none. The nearest VFR residence to the rural MCI site was $11 \mathrm{~km}$ away, although a volunteer first responder was on-scene within 5 minutes (as shown in Table 2). Both the urban and suburban MCI locations had numerous volunteers and ambulance stations within a $10-\mathrm{km}$ radius, while the rural incident took place in a more remote setting, with only 9 VFR residences and no ambulance stations within $10 \mathrm{~km}$.

Response time varied greatly between the $3 \mathrm{MCIs}$, as shown in Tables 3-5. As expected, the urban and suburban MCIs had a greater number of first responders on-scene quickly than did the rural $\mathrm{MCI}$, although one volunteer arrived at the rural $\mathrm{MCI}$ within 5 minutes. VFRs were first on the scene for both the suburban and rural MCIs, and in the urban MCI they arrived within the first 5 minutes together with the ambulance. As shown in Figure 2, the accumulation of VFRs to the scene of the incident was similar to the accumulation of regular MDA ambulances, mobile intensive care units, and specialized MCI response vehicles. In the urban MCI, VFRs accounted for approximately half of the total personnel on-scene. In contrast, in the suburban and rural MCIs the VFR personnel accounted for approximately one-third of the response force.

\section{DISCUSSION}

Previous studies have concluded that volunteers from the community can serve as a vital component of MCI management as they "can and will act as responders." ${ }^{24,25}$ Volunteers can therefore enhance the response capacity of all systems and augment the ability of the community to manage mass casualty incidents and other resource-intensive events. ${ }^{26}$ Training such volunteers in advance facilitates the provision of early warning on the occurrence of an emergency and increases the capacity to provide life-saving services to the afflicted communities. ${ }^{27}$ 
FIGURE 1

\section{Map showing the location of VFRs and ambulances around each mass casualty. The volunteer locations represent place} of residence and the ambulance locations represent station location.

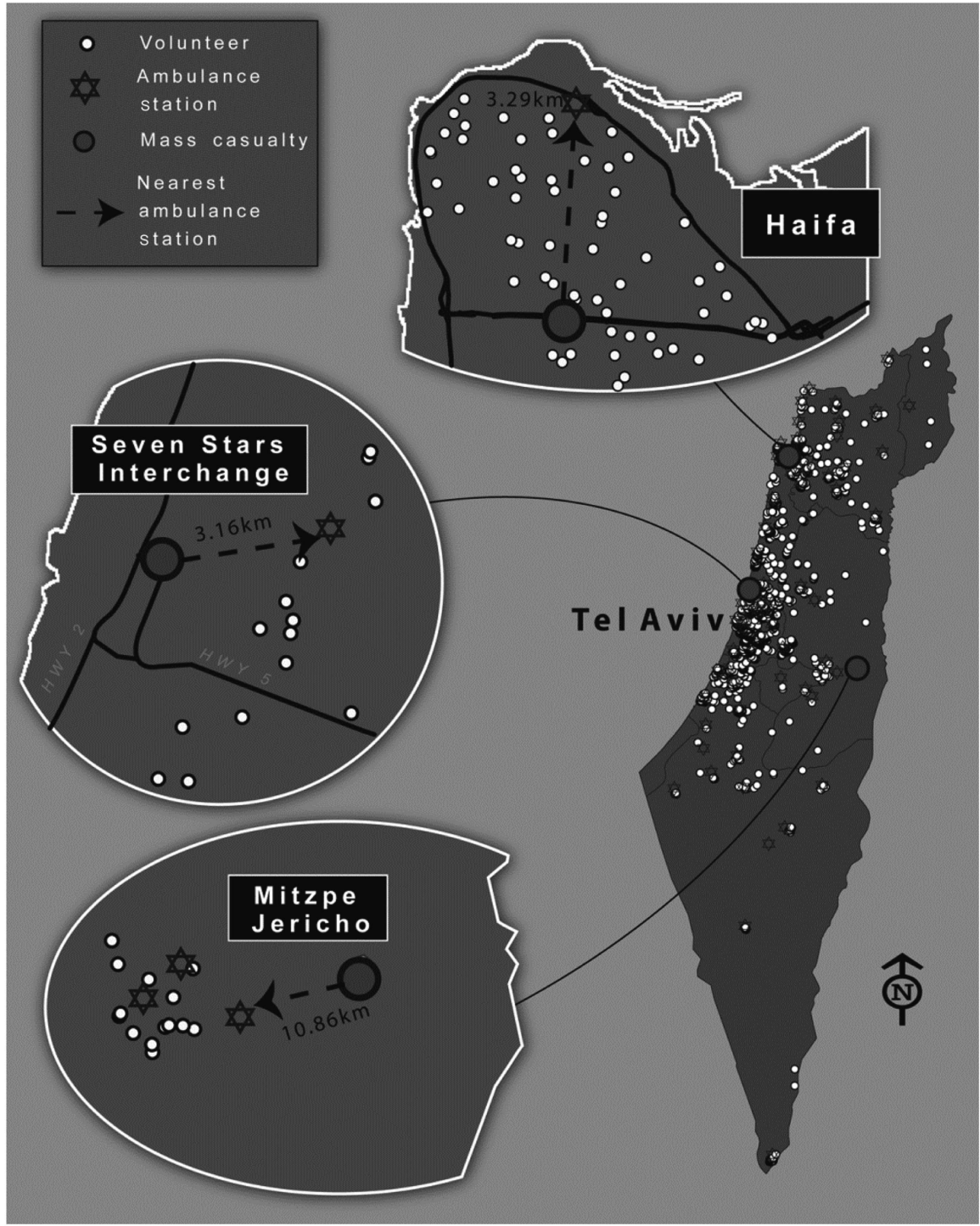

As an integral component of its emergency preparedness for MCIs, the MDA created the model of on-call volunteers that are both well-trained and equipped with basic life-support equipment that enables them to be deployed in parallel to the dispatch of routine on-shift forces. ${ }^{15}$ The results of this study demonstrate that the MDA VFR program has a positive impact on prehospital response times in MCIs. In all 3 incidents examined in this study, VFRs were the first to arrive on-scene. Moreover, the use of VFRs increases the number of on-scene personnel qualified to provide emergency care and assist with triage and evacuation. A common characteristic of MCIs is a lack of vital resources, particularly professional first responders; reinforcement by trained volunteers therefore contributes to a trauma system's capacity to manage a surge of casualties. ${ }^{28,29}$

Effective deployment of volunteers during emergencies requires that they be trained and equipped with relevant competencies and familiarized with the varied threats and characteristics of MCIs. ${ }^{30}$ Ensuring the ability of the VFRs to operate efficiently under the authority of the organization and function effectively within the chain of command necessitates implementation of preparatory measures. In order to ascertain that the VFRs are both competent to provide lifesaving procedures and able to function as integral team 
Number of Volunteer First Responder (VFR) and Magen David Adom (MDA) Personnel On-scene, by Response Time and Role, for the Suburban Mass Casualty Incident (MCI) at the Seven Stars Interchange

No. of Personnel by Response Time

Suburban (Seven Stars)

49 Casualties

MDA Ambulance

Ambulance Intensive Care Unit

$\mathrm{MCl}$ specialists

Cumulative MDA

MDA personnel, \%

VFR VFR

Cumulative VFR

VFR, \%

Cumulative all personnel

All personnel, \%

\begin{tabular}{ccc} 
& & No. of Perso \\
\hline $\mathbf{0 - 5}$ min & $\mathbf{5 - 1 0 ~ m i n}$ & $\mathbf{1 0 - 1 5}$ min \\
& 6 & 8 \\
& 3 & 3 \\
& & \\
0 & 9 & 20 \\
$0 \%$ & $21 \%$ & $48 \%$ \\
3 & 2 & 3 \\
3 & 5 & 8 \\
$18 \%$ & $29 \%$ & $47 \%$ \\
3 & 14 & 28 \\
$5 \%$ & $24 \%$ & $47 \%$
\end{tabular}

$15-20$ min
15
3
38
$90 \%$
6
14
$82 \%$
52
$88 \%$

20-25 min

$25+\min$

2

2

42

$100 \%$

3

17

$100 \%$

59

$100 \%$

\section{TABLE 4}

Number of Volunteer First Responder (VFR) and Magen David Adom (MDA) Personnel On-scene, by Response Time and Role, for the Urban Mass Casualty Incident (MCI) in Haifa

No. of Personnel by Response Time

Urban (Haifa)

51 Casualties

MDA Ambulance

Ambulance Intensive Care Unit

$\mathrm{MCl}$ specialists

Cumulative MDA

MDA personnel, \%

VFR

VFR

Cumulative VFR

VFR, \%

Cumulative all personnel

All personnel, \%

$\begin{array}{ccc}\mathbf{0 - 5} \mathbf{~ m i n} & \mathbf{5 - 1 0} \mathbf{m i n} & \mathbf{1 0 - 1 5} \mathbf{~ i n} \\ 5 & 2 & 2 \\ 5 & & \\ 10 & 12 & 14 \\ 56 \% & 67 \% & 78 \% \\ 11 & 12 & 2 \\ 11 & 23 & 25 \\ 34 \% & 72 \% & 78 \% \\ 21 & 35 & 39 \\ 42 \% & 70 \% & 78 \%\end{array}$

$15-20 \mathrm{~min}$

20-25 min

$25+\min$

17

$94 \%$

3

28

$88 \%$

45

$90 \%$
18

$100 \%$

3

32

$100 \%$

50

$100 \%$

\section{TABLE 5}

Number of VFR and MDA Personnel On-scene, by Response Time and Role, for the Rural Mass Casualty Incident (MCI) in Mitzpe Jericho

No. of Personnel by Response Time

Rural (Mitzpe Jericho)

22 Casualties

$0-5 \min$

$5-10 \min$

$10-15 \min$

$15-20 \mathrm{~min}$

20-25 min

$25+\min$

MDA Ambulance

Ambulance Intensive Care Unit

$\mathrm{MCl}$ specialists

Cumulative MDA

MDA personnel, \%

VFR

Cumulative VFR

VFR, \%

Cumulative all personnel

All personnel, \%

$\begin{array}{ll} & \\ 0 & 0 \\ 0 \% & 0 \% \\ 1 & 1 \\ 1 & 2 \\ 4 \% & 9 \% \\ 1 & 2 \\ 1 \% & 3 \%\end{array}$

1
4
5
$9 \%$
5
7
$30 \%$
12
$16 \%$

3

8
$15 \%$
7
$30 \%$
15
$19 \%$

4

26 
FIGURE 2

Time-To-Arrival on Scene by Responder Type.

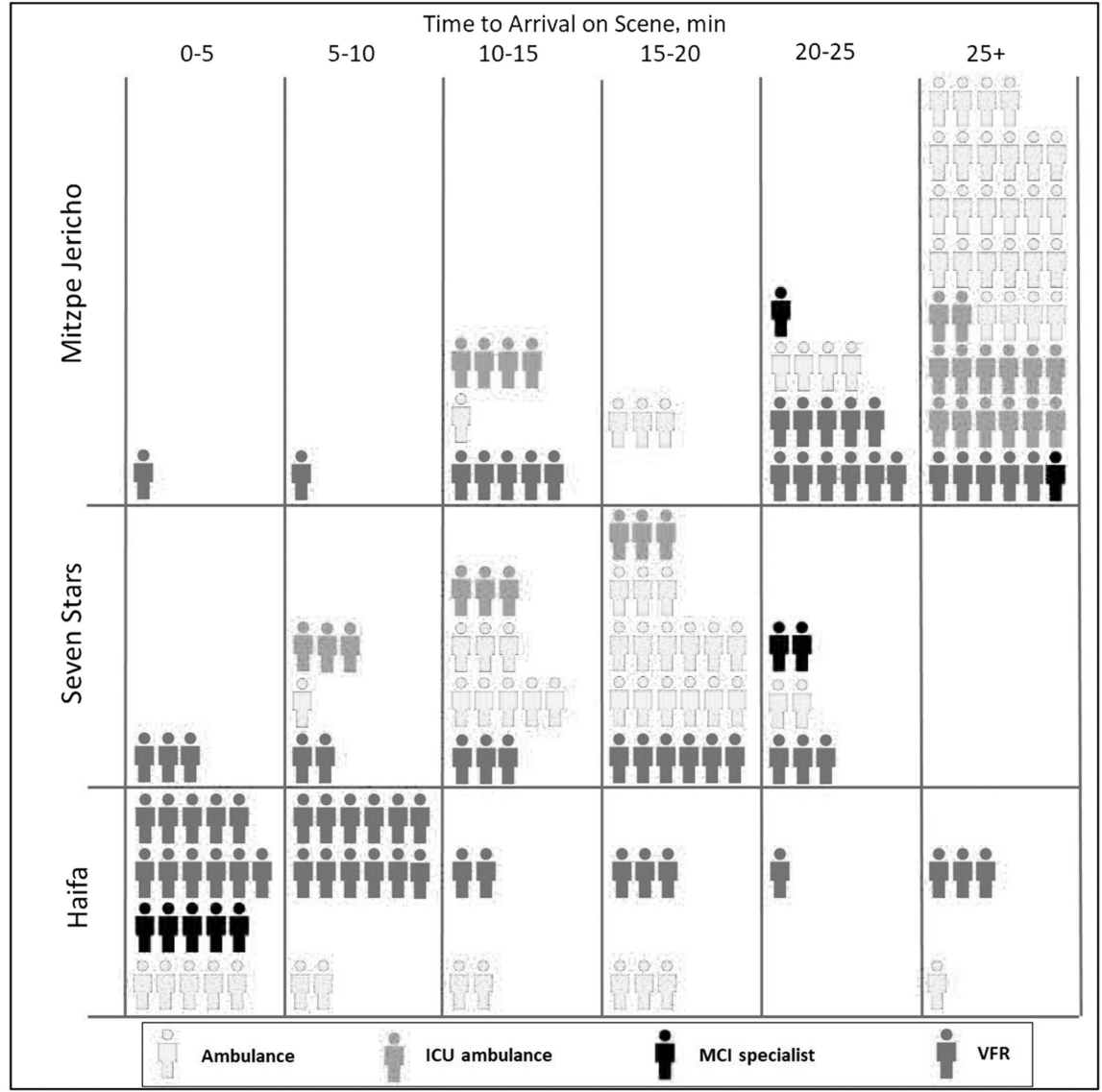

members within the community of first responders, they participate in periodic training programs initiated by the MDA. ${ }^{15}$ These joint training activities, along with routine interaction, enable the VFRs and the ambulance staff to function in a coordinated, collaborative response during MCIs and maintain the organizational structure both on- and off-scene. 22,24

A high degree of training for volunteers enables them to effectively provide emergency care, conduct initial triage, and prepare patients for evacuation to the hospital, allowing MDA ambulances to arrive on-scene and evacuate patients more rapidly. ${ }^{22}$ The existence of qualified volunteers dispersed across the country is instrumental to solidifying the response to MCIs and elevating community resilience and cohesiveness between the MDA, the VFRs, and the communities they serve. ${ }^{31}$ In the case of the MDA, the VFRs are rooted as an integral part of the organization and thus receive ongoing attention, which strengthens their commitment to serve as community responders. ${ }^{22}$ The effective retention of the VFRs over time is evident in their continuous deployment since the program's inception in 2000.
Differences have been highlighted between rural and urban preparedness and abilities to manage varied emergencies. ${ }^{26}$ Capacities of rural communities to respond to MCIs have frequently been reported to be suboptimal, and thus reinforcement of competent medical personnel is of utmost significance. ${ }^{26,29}$ The MCI in the rural area was found to have been particularly impacted by the use of VFRs, as we observed that within 10 minutes of the incident occurring, the only trained personnel at the MCI location were the 2 VFRs. Their rapid arrival ensured that the scene was prepared and preliminary lifesaving actions were taken prior to the ambulances' arrival, enabling a more rapid triage and evacuation process. The perceived advantage in deploying the VFRs is supported by the findings of previous studies that found that volunteers in rural areas can be sustained over time if they are trained and continuously supported by the stakeholders and/or governmental authorities. ${ }^{32}$

Contrary to previous studies suggesting that community volunteers in urban areas may feel unable to make an impact, our results point to the contrary. ${ }^{26}$ In this case, while ambulance personnel and VFRs both arrived quickly to the MCI scene, the VFR personnel were able to provide 
additional critical support, such as secondary life-saving and first aid duties and preparing patients for evacuation; these actions likely improved patient outcomes.

\section{Strengths and Limitations}

This study provides unique insight into the effectiveness of a volunteer first response system. Using detailed temporal data, we were able to discern the role of VFRs in enhancing prehospital care in the event of an MCI in Israel. Additionally, the use of mapping enables the visualization and quantification of VFR and ambulance distributions across the country and around each MCI presented in this study. However, this study mapped VFRs by their place of residence; they may not be at home when called to a nearby incident, though they are required to have first aid and lifesaving equipment at hand. Further and more detailed geographical analysis in the next phase of this study will enable us to identify key locations for VFR recruitment and ambulance stand-by.

While the use of MCIs from multiple time periods may facilitate a simple longitudinal analysis, the differing characteristics between events (urban, suburban, and rural) inhibit any meaningful inference from a temporal comparison. Since the program's inception, the number of VFRs has increased, reducing overall response times and increasing the number of available personnel. Ongoing analysis of MCIs is therefore important to ensure that volunteer recruitment and deployment is optimal.

These data provide preliminary insight into the importance of VFRs and their geographical distribution. Not only should resources mirror the population distribution across the map, but places of work, recreation, and transportation should also be taken into account in their planning. As this study focused on 3 bus rollover incidents, our findings underscore the importance of resource availability in major transit corridors. Future analyses will consider the distribution of prior MCIs when prescribing suitable locations for first response resource allocation (eg, VFR recruitment).

\section{Future Research}

As this study demonstrated, the role of VFRs is critical in providing a quicker response during MCIs. A sufficient number of additional qualified first responders able to reach the scene of an MCI in a timely manner is key to the success of this program. However, as shown in the map and geographical analysis, the spatial distribution of VFRs is crucial to rapid response. Future research should therefore focus on optimizing the distribution of VFRs across the whole country according to criteria such as population density or potential $\mathrm{MCI}$ occurrence. This optimization will result in more effective delivery of care and reductions in VFR program costs.

\section{CONCLUSIONS}

The VFR program provides an important and effective life-saving resource to supplement emergency first response in Israel. Drawing upon volunteers distributed across the country, VFRs were able to arrive at the scene of each of the $3 \mathrm{MCIs}$ prior to or simultaneously with ambulance personnel. Given the known importance of rapid response to trauma, VFRs likely contribute to reduced trauma mortality, although further research will examine this question specifically.

The use of volunteer first responders may be most effective in rural and remote regions globally. While we focused exclusively on MCIs, VFRs provide a valuable resource for emergency medical response in general, and their use outside of Israel is recommended on the basis of our findings.

\section{About the Authors}

Volunteers, Public Relations, Training, Fund-raising E International Relations Division, Magen David Adom, Tel-Aviv, Israel (Dr Yafe); Geographisches Institut, Humboldt-Universität zu Berlin, Berlin, Germany (Dr Walker); Department of Geography, Simon Fraser University, Burnaby, British Columbia, Canada (Dr Walker); Department of Nutrition and Exercise Physiology, Elson S. Floyd College of Medicine, Washington State University, Spokane, Washington (Dr Amram); School of Population and Public Health, University of British Columbia, Vancouver, British Columbia, Canada (Ms Randall); Department of Public Health, Ben Gurion University of the Negev, Be'er Sheva, Israel (Dr Friger); Department of Disaster Management $\mathcal{E}$ Injury Prevention, School of Public Health, Faculty of Medicine, Tel Aviv University, Israel (Dr Adini).

Correspondence and reprint requests to Bruria Adini, Hatamar 16, Bat Chen, 40290, Israel (e-mail: adini@tauex.tau.ac.il).

\section{Acknowledgements}

The authors would like to thank the numerous volunteers that contribute their time, energy, and personal (mental) resources to help others in need.

\section{Competing Interests}

Dr Eli Yafe is director of the MDA VFR program. The authors state that they have no other competing interests. The authors alone are responsible for the content and writing of the paper.

\section{Sources of support}

No funding was requested nor provided for this study.

\section{REFERENCES}

1. Shoher A, Chang DC, Efron D, et al. Multiple, simultaneous trauma patients: are they worse off? J Trauma Inj Infect Crit Care. 2006;61: 611-615.

2. Hammond J. Mass casualty incidents: planning implications for trauma care. Helsinki, Finland. Scand J Surg. 2005;94:267-271.

3. Lennquist S. Management of major accidents and disasters: an important responsibility for the trauma surgeons. J Trauma Inj Infect Crit Care. 2007;62:1321-1329.

4. Committee on Trauma, American College of Surgeons. Resources for Optimal Care of the Injured Patient 2006. Chicago, IL: American College of Surgeons; 2006.

5. Levi L, Michaelson M, Admi H, et al. National strategy for mass casualty situations and its effects on the hospital. Prehosp Disaster Med. 2002;17: 12-16.

6. Trauma System Agenda for the Future: Key Issues in Developing Inclusive Trauma Systems. http://www.nhtsa.gov/people/injury/ems/ emstraumasystem03/intro-keyissue.htm. Accessed February 10, 2017.

7. Eiseman BMD. Combat casualty management for tomorrow's battlefield: urban terrorism. J Trauma Inj Infect Crit Care. 2001;51(5):821-823. 
8. Halpern P. Mass-casualty, terrorist bombings: implications for emergency department and hospital emergency response (Part II). Prehosp Disaster Med. 2003;18:235-241.

9. Jenkins J, McCarthy M, Sauer L, et al. Mass-casualty triage: time for an evidence-based approach. Prehosp Disaster Med. 2008;23:3-8.

10. Iserson KV, Moskop JC. Triage in medicine, Part I: Concept, history, and types. Ann Emerg Med. 2007;49:275-281.

11. Kennedy K, Aghababian RV, Gans L, Lewis CP. Triage: techniques and applications in decisionmaking. Ann Emerg Med. 1996;28:136-144.

12. Aylwin CJ, König TC, Brennan NW, et al. Reduction in critical mortality in urban mass casualty incidents: analysis of triage, surge, and resource use after the London bombings on July 7, 2005. Lancet. 2006;368:2219-2225.

13. Soffer D, Klausner JM. Trauma system configurations in other countries: the Israeli model. Surg Clin North Am. 2012;92:1025-1040.

14. First Responders. https://www.mdais.org/en/first-responders. Accessed February 10, 2017.

15. Alpert EA, Lipsky AM, Elie ND, et al. The contribution of on-call, volunteer first responders to mass-casualty terrorist attacks in Israel. Am J Disaster Med. 2015;10:35-39.

16 Statement on Disaster and Mass Casualty Management. http://www. facs.org/. Accessed February 10, 2017.

17. Ryan J, Sibson J, Howell G. Assessing injury severity during general war. Will the Military Triage system meet future needs? J R Army Med Corps. 1990;136:27-35.

18. Nathens A, Maier R, Brundage $S$, et al. The effect of inter-facility transfer on outcome in an urban trauma system. J Trauma Inj Infect Crit Care. 2003;55:444-449.

19. Davis D, Poste J, Hicks T, et al. Hospital bed surge capacity in the event of a mass-casualty incident. Prehosp Disaster Med. 2005;20:169-176.

20. Mistovich JJ, Hafen BQ, Karren KJ, et al. Prehospital Emergency Care. Boston: Brady Prentice Hall Health; 2004.

21. Feliciano DV, Mattox KL, Moore EM. eds. Trauma, 6th ed. New York: McGraw-Hill Professional; 2008.
22. Shemer J, Shapira S. Establishing of a nationwide trauma system in Israel. Harefuah. 1995;129:526-529.

23. Ramesh AC, Kumar S. Triage, monitoring, and treatment of mass casualty events involving chemical, biological, radiological, or nuclear agents. J Pharm Bioallied Sci. 2010;2:239.

24. Jacobs LM Jr. Joint committee to create a national policy to enhance survivability from mass casualty shooting events: Hartford Consensus II. J Am Coll Surg. 2014;218:476-478.

25. Goolsby C, Branting A, Chen E, et al. Just-in-time to save lives: a pilot study of layperson tourniquet application. Acad Emerg Med. 2015;22: 1113-1117.

26. Flint CG, Stevenson J. Building community disaster preparedness with volunteers: Community Emergency Response Teams in Illinois. Nat Hazards Rev. 2009;11:118-124.

27. Susetyo Koespradiyanto E, Adinugroho K. Community based emergency preparedness as key part in anticipation mass casualties in surrounding oil and gas industry. Paper presented at: International Conference on Health, Safety and Environment in Oil and Gas Exploration and Production; September 11-13, 2012; Perth, Australia.

28. Furbee PM, Coben JH, Smyth SK, et al. Realities of rural emergency medical services disaster preparedness. Prehosp Disaster Med. 2006;21: 64-70.

29. Glow SD, Colucci VJ, Allington DR, et al. Managing multiple-casualty incidents: a rural medical preparedness training assessment. Prehosp Disaster Med. 2013;28:334-341.

30. Hamilton SE. Volunteers in disaster response: The American Red Cross. J Aggress Maltreat Trauma. 2005;10:621-632.

31. Aldrich DP, Meyer MA. Social capital and community resilience. Am Behav Sci. 2015;59(2):254-269.

32. Farmer J, Currie M, Kenny A, et al. An exploration of the longer-term impacts of community participation in rural health services design. Soc Sci Med. 2015;141:64-71. 